\title{
AN IMPROVED TROPOSPHERIC TOMOGRAPHY METHOD BASED ON THE DYNAMIC NODE PARAMETERIZED ALGORITHM
}

\author{
Wenyuan ZHANG ${ }^{1,2)}$, Shubi ZHANG ${ }^{1,2)}$ *, Nan DING ${ }^{3)}$ and Pengxu MA ${ }^{1,2)}$ \\ 1) School of Environment Science and Spatial Informatics, China University of Mining and Technology, Xuzhou 221116, China \\ 2) NASG Key Laboratory of Land Environment and Disaster Monitoring, China University of Mining and Technology, Xuzhou 221116, China \\ ${ }^{3)}$ School of Geography, Geomatics and Planning, Jiangsu Normal University, Xuzhou 221116, China
}

*Corresponding author's e-mail: zhangsbi@163.com

\begin{tabular}{l} 
ARTICLE INFO \\
\hline Article history: \\
Received 12 October 2019 \\
Accepted 19 March 2020 \\
Available online 4 May 2020 \\
\hline
\end{tabular}

\section{Keywords:}

GNSS meteorology

Water vapor tomography

Adaptive node parameterized

Spline interpolation

Radiosonde

\begin{abstract}
In the general tropospheric tomography model, the tomographic area is divided into a large number of voxels, which provides convenience for reconstructing tomographic observation equations. However, due to the defect of GNSS acquisition geometry, there are plenty of empty voxels for any tomographic epoch. Moreover, an unreasonable assumption that water vapor density is constant within a voxel was imposed on the tomographic model. In this study, we proposed an improved method based on the dynamic node parameterized algorithm to solve both key problems. The proposed approach first tries to select effective GNSS signals and determines the dynamic scope of the tomographic area using the dynamic algorithm. The parameterization of the tomography model is performed by a cubic spline formula and Gauss weighted function. Additionally, a piecewise linear fitting method based on Newton-Cotes interpolation is introduced to estimate the tomographic observation of slant water vapor (SWV). The experimental results show that the average number of effective signals increased by $32.33 \%$ and the mean RMSE of the tomographic results is decreased by $45 \%$ with the proposed method. Further, compared with the tomographic results of the general method, the improved solutions have a more centralized distribution and a smaller bias.
\end{abstract}

\section{INTRODUCTION}

Three-dimensional (3D) tropospheric water vapor, characterized by dynamic and variable, plays a key role in atmospheric modeling and weather forecasting (Benevides et al., 2015; Zus, 2018). Water vapor observations with high spatiotemporal resolution and high-quality is useful to relieve the adverse effects of extreme weather events (Andersson et al., 2007). GNSS tomography, retrieving the local 3D water vapor field, has bloomed into an efficient atmospheric water vapor detection technique to study rainstorm events and climatic change (Chen et al., 2017; Wang et al., 2018). Bevis et al. (1992) first presented that water vapor could be estimated by the Global Positioning System (GPS), and tomography technology was introduced to GPS water vapor research in 2000 to offset the lack of two-dimensional water vapor information (Flores et al., 2000). Since then, considerable research and experiments have confirmed that GNSS tomography performs well compared with balloon soundings, water vapor radiometer, radar detection, infrared microwave radiometer, and other technologies. Additionally, in the study of Chen and Liu (2014), it is noticeable that tomography deviates less than to radiosonde observations and numerical weather models.
The 3D water vapor field retrieved by GNSS tomography not only supplies water vapor density at different locations in the horizontal direction but also provides water vapor profile information at different altitudes. However, there are still some limitations including distribution of GNSS stations, the topological structure of satellite constellations, and the quality of observation signals, which decrease the accuracy of tomographic solutions. In view of the geometric confinement of GNSS satellite reference station networks and satellite constellations, the satellite signal is an inverted cone that is mostly concentrated in the middle and upper layer of the tomographic region (Zhao et al., 2019b). These structural geometries cause a mix-determined problem in that a large number of voxels are not passed through by any ray while excessive rays may pass from one voxel (Rohm, 2013), which results in ill-conditioned equations and a rank-deficient matrix (i.e., there are many zero elements in the coefficient matrix of the observation equation) when constructing tomographic observation equations. Several methods have been proposed in attempts to resolve this ill-conditioned problem. It is a given that (1) horizontal and vertical constraints are added and optimized (Flores et al., 2000; Song et al., 2006); (2) a priori information, which is initialized and updated by radiosonde, the European 
Centre for Medium-Range Weather Forecasts (ECMWF) and Atmospheric Infrared Sounder (AIRS), are employed in GNSS tomography (Yao and Zhao, 2016; Benevides et al., 2018); (3) observations from GPS, BDS, GALILEO and GLONASS are used in the GNSS tomography model (Benevides et al., 2017; Dong and Jin, 2018; Zhao et al., 2018a; Zhao et al., 2019a); (4) the tomographic area is expanded to increase the number of available observations (Ding et al., 2018b; Zhao et al., 2018c; Yang et al., 2019); and (5) data in addition to GNSS observations (e.g., InSAR and GNSS-R) are used to add constraint information (Benevides et al., 2016; Heublein et al., 2019; Jaberi Shafei and Mashhadi-Hossainali, 2018). Consequently, the quantity of the blank voxels is reduced, and the water vapor density of blank voxels can be estimated by additional constraints, which minimizes the rank defect of the observation matrix and improves the accuracy of the tomographic results. However, in most existing studies, a fixed box-shaped area is preset according to the location of the GNSS stations, which represents the final 3D water vapor tomography area for any tomographic epoch. Then GNSS signals passing from the top boundary of the tomography area are considered as effective signals, and all rays penetrating from the side of tomography area are eliminated without considering their usefulness. This behavior neglects useful observations and aggravates the ill-posed of water vapor tomography system.

The voxel discretization error caused by unreasonable assumption also is researched in some studies. Perler et al. (2011) proposed that the water vapor density of the voxel be determined by a weighted sum of the 8 water vapor density values at the corners of the voxel instead of the water vapor density at the voxel center. Besides, a new parameterized method based on inverse distance weighted (IDW) interpolation introduced by (Ding et al., 2017) and (Zhao et al., 2018b) showed a horizontal parameterized approach without discretization in the horizontal direction. However, these improved parameterized methods based on the voxel model do not completely avoid the problem of discontinuity. Ding et al. (2018b) introduce a node discretization method with the novel convex hull boundaries, which is improved in this paper.

To construct a matching tomographic area and retrieve a continuous $3 \mathrm{D}$ water vapor field, this paper proposes an improved water vapor tomographic method. Contrary to obtaining the tomographic region according to the location distribution of GNSS stations in the traditional model, the optimized tomography model gives priority to the spatial distribution of signals in each layer when determining the boundary and node position of the tomographic field. It should be noted that the tomography model would be dynamic as a result of the constant changing of the distribution of signals. In addition, the tomographic area is discretized into nodes instead of voxels with the node parameterized algorithm. This paper is structured as follows. Section 2 describes the GNSS processing procedure. Section 3 provides details about the improved node parameterized method. Experiments are performed and results are discussed in Section 4. Conclusions are drawn in Section 5.

\section{GNSS TOMOGRAPHY DATA PREPROCESSING}

In the process of positioning with GNSS, there are many errors including ionospheric delays, tropospheric delays, and multipath effects, which are the troubles when people use GNSS for precise positioning, precise navigation, and precise timing (Zumberge et al., 1997; Lu et al., 2015). However, in view of GNSS meteorology, the tropospheric delay induced by water vapor is considered as critical information for retrieving 3D wet refractivity (WR)/water vapor density (WVD). Therefore, the high-accuracy tropospheric delay is necessary to support water vapor tomography.

The GNSS electromagnetic wave signal is influenced by the atmosphere on the way transmitted from satellites to receivers, resulting in the GNSS signals delay including ionospheric delay and tropospheric delay (Möller and Landskron, 2019). GNSS combining dual-frequency observations is used to eliminate the ionospheric delay, while the remaining one is estimated as an unknown parameter using empirical tropospheric models (Flores et al., 2001).

The tropospheric delay (ZTD) usually refers to the total delay in the zenith direction of the GNSS station, which includes the zenith hydrostatic delay (ZHD) and zenith wet delay (ZWD). The relationship is shown in Equation (1).

$\mathrm{ZTD}=\mathrm{ZHD}+\mathrm{ZWD}$,

ZWD which contains water vapor information usually be extracted from ZTD by eliminating ZHD. Currently, the main methods of obtaining ZTD are double-difference method and precise point positioning (PPP) method (Alber et al., 2000; Yu et al., 2018b). Some empirical tropospheric delay models (e.g., Saastamoinen model, Hopfield model, and Black model) are used to estimate ZHD values.

As mentioned above, ZWD only represents the wet delay values in the zenith direction of the station, which induces a defect that the 3-D water vapor field of a tomographic area cannot be fully retrieved. Therefore, with the assistant of wet mapping function and wet delay gradients, ZWD can be converted into slant wet delay (SWD) which represents the wet delay information in the direction of the GNSS signals.

$$
\begin{aligned}
\mathrm{SWD}= & m^{w}(e) \cdot Z W D+m_{g}^{w}(e) \cdot \\
& \cdot\left(G_{N S}^{w} \cdot \cos (\varphi)+G_{E W}^{w} \cdot \sin (\varphi)\right)+R,
\end{aligned}
$$

where $G_{N S}^{w}$ and $G_{E W}^{w}$ are the wet gradient delay parameters, $m^{w}(e)$ and $m_{g}^{w}(e)$ represent the wet mapping function and horizontal gradient function, respectively. $e$ and $\varphi$ are the elevation and azimuth, 


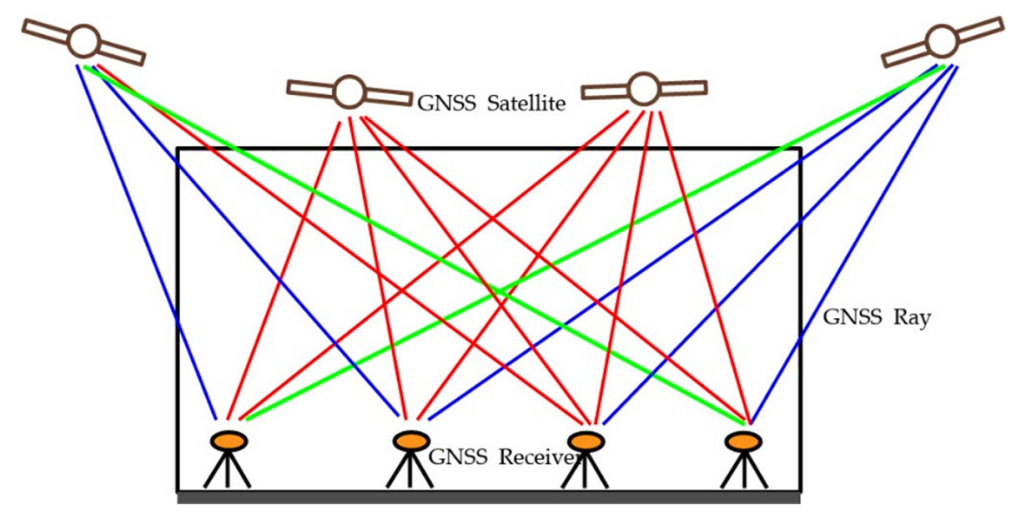

Fig. 1 Observing geometry of GNSS satellites and ground-based receiver. The black frame represents the boundary of the tomographic area, the red lines, blue lines, and green lines show the GNSS electromagnetic signals.

respectively. In this paper, Saastamoinen model was set as the expressions for the ZHD (Saastamoinen, 1972), and VMF1 mapping function was used for calculating the SWDs (Boehm and Schuh, 2004). As far as the gradient components are concerned, the total gradients, similar to the total delays, consist of hydrostatic gradient component and wet gradient one. The former, can be estimated with the surface pressure around each GNSS station (Brenot et al., 2019). Therefore, the latter can be expressed by the difference of the hydrostatic to the total component. $\mathrm{R}$ is the postfit residuals calculated by GAMIT (v10.6) software, and the residuals exceeding 2.5 times the standard deviation were removed (Ding et al., 2018a).

Usually, SWD is used as GNSS tomographic observations to restructure the $3 \mathrm{D}$ WR. In this paper, the slant water vapor (SWV) which has a nearly proportional relationship with the SWD is selected as input information for retrieving the $3 \mathrm{D}$ water vapor field in the node tomographic model.

$\mathrm{SWV}=\Pi \cdot \mathrm{SWD}$,

where $\Pi$ represents the conversion coefficient closely related to the weighted mean temperature of the atmosphere (Zhao et al., 2018b).

\section{DYNAMIC NODE PARAMETERIZED FOR PROPOSED IMPROVED METHOD}

Differ from the construction process of the traditional approach, useful GNSS signals are selected according to elevation angle in the improved method at first, the dynamic tomography area is determined by the geometric structure of signals. This section is organized as follows: Section 3.1 describes the way of selecting effective GNSS signals and determining the tomography area. The detailed process of the node parameterized algorithm for tropospheric tomography is discussed in Section 3.2. In addition, the Algebraic Reconstruction Technique (ART) for solving the rank defect tomographic observation equations is introduced in Section 3.3.

\subsection{OPTIMIZED PREPROCESSING OF GNSS RAYS WITH DYNAMIC ALGORITHM}

In the general GNSS tomography model, as shown in Figure 2a, a fixed cuboid area surrounded by a black border is preset according to the location of the GNSS stations, which represents the final 3-D water vapor tomographic region. However, it is a conspicuous disadvantage that a great number of voxels at the bottom boundary are not signaled. Many useless unknown parameters (i.e., the water vapor density parameter of the empty voxel) have to be estimated by the tomographic model, which reduces the accuracy of tomography model solutions.

\subsubsection{SELECTION OF EFFECTIVE SIGNALS}

Due to the effect of atmospheric bending, the propagation path of GNSS electromagnetic wave signal at low elevation angle $\left(e<15^{\circ}\right)$ bends when it passes through the atmosphere, and the study of (Möller and Landskron, 2019) develop a mixed raytracing method to correct the low elevation observations. In voxel tomography model, tomography area is empirically preseted and signals passing from the top boundary of the tomography area are considered as effective signals (red lines in Fig. 1). However, there is an obvious drawback that same signals with an elevation angle greater than $15^{\circ}$ (blue lines in Fig. 1) are eliminated because they pass through the side of the tomography area. The spatial distribution of GNSS signals changes at different epochs, this behavior will miss some useful observation information and decrease the utilization ratio of effective signals. To make matters worse, a few of signals are regarded as useful signals as they pass through the tomography top (green lines in Fig. 1), and their bending effect caused by the elevation angle below $15^{\circ}$ are ignored. This phenomenon will get worse as the tomographic area gets larger.

To solve this problem, a new method of signal selection based on the elevation angle is proposed. GNSS signals with an elevation angle greater than $15^{\circ}$ are considered as effective observations to retrieve the 


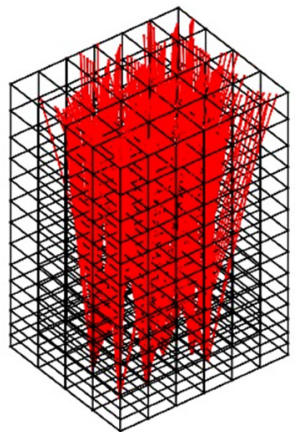

(a)

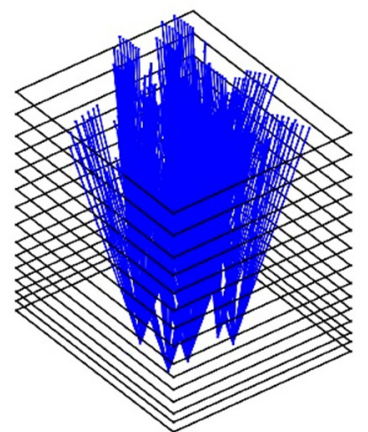

(b)

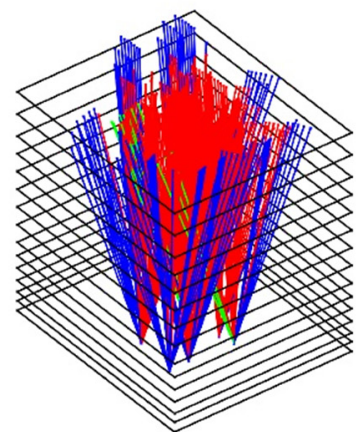

(c)

Fig. 2 The spatial distribution of GNSS signals derived from the conventional approach (a) and the new proposed approach (b) and the comparison of two approaches is shown in (c).

3D water vapor fields in this work. Due to the significant atmosphere bending effect, signals with elevation angle below $15^{\circ}$ are passed. The number of useful signals and the elevation angle of these signals are analyzed on the basis of the 12 GNSS stations in Hong Kong (Fig. 8). Figure 2 shows the spatial distribution of the effective observed signals obtained by the two signal selection methods. It should be noted that the new signal selection method only depends on the elevation angle information of the signals.

A better comparison is shown in Figure 2c: the red lines represent GNSS signals that puncture the tomography top and have the peculiarity of elevation angle greater than $15^{\circ}$; rays with elevation angle below $15^{\circ}$ are indicated by green lines. The blue lines denote the GNSS rays passing through the side of the voxel tomography model but with an elevation angle greater than $15^{\circ}$, which are considered as useful signals in the new selection method. It is shown in Figure $2 \mathrm{c}$ that many signals, especially those penetrating from the side face of the tomography area are introduced to the optimized tomography method, which may improve the performance of tomographic model.

\subsubsection{DETERMINATION OF TOMOGRAPHY AREA}

After selecting useful signals, we determine the tomography boundary according to the spatial position information of GNSS signals. The detailed steps of determining the tomography area are given below:

1. Computing the space position coordinates of high-quality GNSS observation signals, which is very important for determining the location of the tomographic region.

2. Calculating the coordinates of the puncture points (black intersection of the signal and each level in Figure 3a) in each layer.

3. Acquiring the convex hulls covering all puncture points using Graham scan (Graham, 1972; Ding et al., 2018b).

4. Determining the optimal rectangles which are best similarity with the convex hulls using the searching algorithm. The word "similarity", equal to the ratio of convex hull area to rectangle area, is defined to express the similarity between the convex hulls and the rectangles. This rectangle is considered as the optimal tomographic boundaries in this paper.

$\gamma=\frac{S_{\text {convex }}}{S_{\text {rectangle }}} \times 100 \%$,

where $\gamma$ is the similarity, and $S_{\text {convex }}$ as well as $S_{\text {rectangle }}$ represent the area of convex hulls and rectangles, respectively. The procedure of the searching algorithm is as below. An edge of a convex polygon can determine a rectangle parallel to this edge. Thus, a convex polygon can produce many rectangles. The similarity between these rectangles and this convex polygon is calculated in turn, and the rectangle with the highest similarity is chosen as the optimal rectangle boundary.

The green wireframes in Figure $3 \mathrm{a}$ shows the optimized boundary of each layer, compared with the common voxel model boundary (black wireframes in Fig. 2a), the new tomographic model boundary is obvious more suitable for the distribution of GNSS signals. In addition, this new method ensures that the boundary of each layer is consistent with the signal distribution and is dynamic in different epochs. More signal lines are accepted in the node model, which enlarges the scope of the tomographic area and improves the stability of tomographic model.

\subsection{STRATEGY FOR NODE PARAMETERIZATION}

After determining the boundary of the tomographic area, the primary mission of the node parameterized algorithm is to reasonably discretize the tomographic region using nodes. Additionally, the quantitative relationships between the water vapor density of nodes and the SWV of GNSS signals are established according to the spatial location, which is the basis of constructing tomographic observation equations.

\subsubsection{DISCRETIZATION OF TOMOGRAPHIC AREA}

One customarily assumes that the water vapor density is equal everywhere in a small cuboid cell in the general tomographic model. Although it is convenient for reconstructing the 3D water vapor field, this discretized method breaks the continuity of water vapor density in space. Therefore, each voxel in the traditional model is independent and unrelated, 


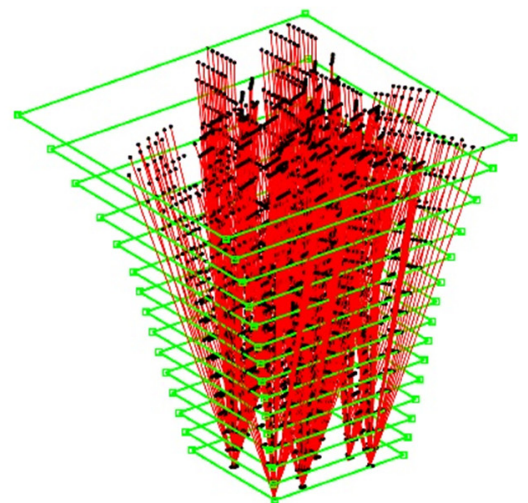

(a)

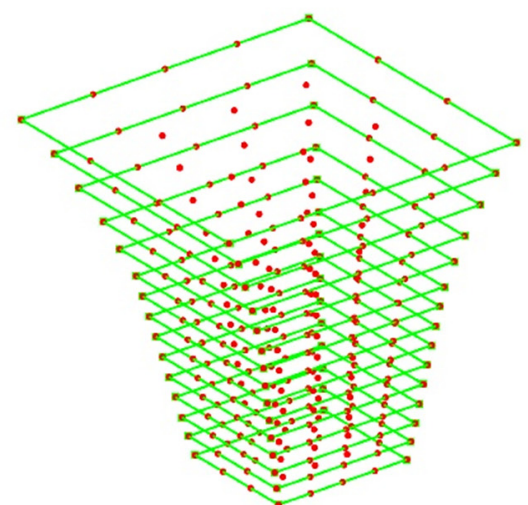

(b)

Fig. 3 (a) Dynamic boundary (green wireframe) on each horizontal level of the improved tomography model, the black point represents the intersection of the signal line and each layer of the tomographic model. (b) Node parameterization approach for GNSS tomography; the red dots are the nodes, and water vapor parameters at the nodes are solved in the tomographic model.

which is no longer match with the actual water vapor distribution. Besides, due to the restrictions on voxel discretization, the SWV value obtained from the voxel tomographic model is different from the actual SWV value of the signal, which is called "discretization error".

As a result, a new discretized method based on nodes is introduced to minimize the discretized error and ensure the continuous spatial characteristics of water vapor. In node discretization, nodes are introduced as discretized units into the construction of a 3D water vapor tomographic field. About the strategy for assigning number and location of horizontal nodes, two options are proposed in this paper.

The first one is that the horizontal distance between nodes (i.e., horizontal resolution) is set at first, this resolution can be equal to that of the voxel model (Perler et al., 2011; Ding et al., 2017). The number and location of horizontal nodes are determined according to the optimal tomographic boundary and the node horizontal resolution. Consequently, node model has less unknown parameters than the voxel model, which is conducive to improve the accuracy of parameter solutions.

The second one is that the number of horizontal nodes is determined in advance. The same number of nodes in each layer generates that the horizontal resolution of node model increases gradually as the altitude decreases and approaches maximum in the surface, which is is consistent with the vertical variation of water vapor density. Furthermore, in the lower troposphere, the node model retrieves higher resolution 3D water vapor field than the voxel model, which is useful for atmospheric modeling.

Obviously, the proposed node discretization method is more consistent with the spatial distribution of water vapor and reduces discretized error. The second scheme is selected in this paper. As shown in Figure $3 \mathrm{~b}$, the tomographic region is divided into red nodes, and the water vapor density of the red nodes is the unknown parameter to be solved.

\subsubsection{REDUCING DISCRETIZED ERROR USING A NUMERICAL INTEGRATION METHOD}

In the classical tomographic model, the total slant water vapor (SWV) of the GNSS signal is equal to the sum of the SWV value of the voxels through which this signal passes; the product of the length of signal in the voxel and the water vapor density of the voxel is regarded as the SWV value of the voxel (Flores et al., 2000). As noted above, this computed method brings large discretization error to tomographic observation equations.

To reduce discretized error, a numerical integration method based on the Newton-Cotes interpolation formula is adopted in the node parameterization. The SWV value along the radio signal from satellite antenna $(q)$ to receiver antenna $(r)$ can be expressed by Equation (5),

$S W V_{q}^{r}=10^{-6} \cdot \int_{q}^{r} \rho(s) d s$,

where $s$ represents the signal path from satellite antenna $(q)$ to receiver antenna $(r)$, and $\rho(s)$ is the water vapor density along the signal. The integral formula is regularly encountered in mathematics. However, the conventional quadrature formula cannot be used, as the integrand function $\rho(s)$ cannot be expressed by an elementary function. The numerical integration method based on the idea of numerical approximation is usually used to solve such problems. The Cotes formula (6) and the complex Cotes formula (7) are expressed as follows:

$$
\begin{gathered}
\int_{a}^{b} f(x) \approx \frac{b-a}{90} \cdot\left[\begin{array}{r}
{\left[f\left(x_{0}\right)+32 f\left(x_{1}\right)+12 f\left(x_{2}\right)+\right.} \\
\left.+32 f\left(x_{3}\right)+7 f\left(x_{4}\right)\right],
\end{array}\right. \\
x_{i}=a+\frac{i \cdot(b-a)}{4}, \quad i=0,1,2,3,4 .
\end{gathered}
$$



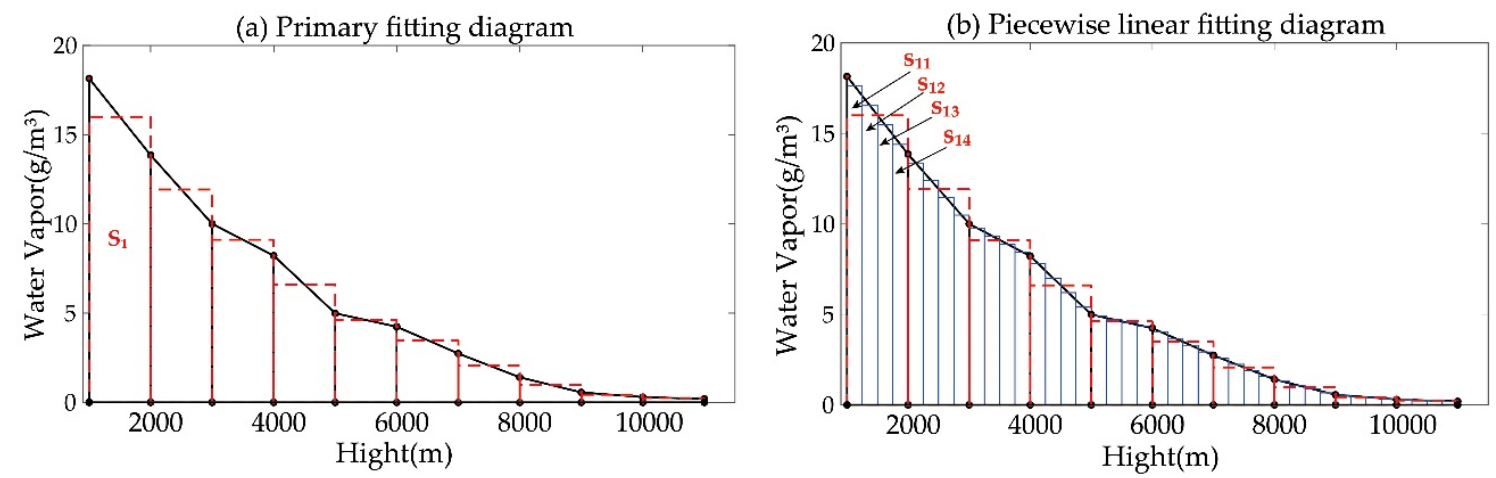

Fig.4 (a) Integral schematic diagram of water vapor density based on linear fitting. (b) Integral schematic diagram of water vapor density based on piecewise linear fitting.

$$
\begin{aligned}
& \int_{a}^{b} f(x) \approx \sum_{i=0}^{n-1} \frac{h}{90} \cdot\left[7 f\left(x_{i}\right)+32 f\left(x_{i+\frac{1}{4}}\right)\right. \\
& +12 f\left(x_{i+\frac{1}{2}}\right)+32 f\left(x_{i+\frac{3}{4}}\right) \\
& \left.+7 f\left(x_{i+1}\right)\right] \\
& \approx \frac{h}{90} \cdot\left[7 f(a)+32 f \sum_{i=0}^{n-1}\left(x_{i+\frac{1}{4}}\right)+\right. \\
& +12 f \sum_{i=0}^{n-1}\left(x_{i+\frac{1}{2}}\right)+32 f \sum_{i=0}^{n-1}\left(x_{i+\frac{3}{4}}\right)+ \\
& \left.+14 f \sum_{i=0}^{n-1}\left(x_{i}\right)+7 f(b)\right] \text {, } \\
& x_{i+\frac{1}{4}}=x_{i}+\frac{(b-a)}{4 n}, x_{i+\frac{1}{2}}=x_{i}+\frac{(b-a)}{2 n} \text {, } \\
& x_{i+\frac{3}{4}}=x_{i}+\frac{3(b-a)}{4 n}, \quad i=0,1,2,3, \cdots, n-1 .
\end{aligned}
$$

where $[a, b]$ is a limited interval, equivalent to the distance from the top of the troposphere to the receiver antenna in this paper; $x_{i}, x_{i+\frac{1}{4}}, x_{i+\frac{1}{2}}, x_{i+\frac{3}{4}}$ are interpolation points; and $n$ represents the number of interpolation points in each small interval.

The specific application of the formulas is as follows: As described by Figure 4a, the SWV value of a signal corresponds to the area enclosed by the whole curve and coordinate axis. The solutions to the problem mentioned above is to divide the general voxel model into two small steps: The first step is computing the product of the length of the signal passing through the voxel and the water vapor density of this voxel, which corresponds to the area of a small red rectangle in Figure 4a (e.g., $S_{1}$ ). The SWV of all voxels passed by the signal are added together as the total SWV value of the signal is the second step, which corresponds to the sum of $n$ small red rectangular areas (e.g., $S_{1}+S_{2}+\cdots+S_{n}$ ). It should be noted that Figure 4 is only a schematic diagram to illustrate the principle of numerical integration fitting. Equation (8) shows this calculation method with mathematical formulas.

$$
\begin{gathered}
S W V_{q}^{r}=10^{-6} \cdot \int_{a}^{b} f(s) d s \approx 10^{-6} \cdot\left[\rho_{1} \cdot \frac{\Delta d_{1}}{\sin (e)}+\right. \\
\left.+\rho_{2} \cdot \frac{\Delta d_{2}}{\sin (e)}+\cdots+\rho_{i} \cdot \frac{\Delta d_{i}}{\sin (e)}+\cdots+\rho_{n} \cdot \frac{\Delta d_{n}}{\sin (e)}\right]
\end{gathered}
$$

where $\Delta d_{i}$ is the vertical distance of the signal passing through a voxel, which corresponds to the width of the small red rectangle in Figure 4a; $\rho_{i}$ is the water vapor density in this voxel, which corresponds to the height of the small red rectangle; and $e$ represents the elevation angle of the satellite signal.

From a mathematical point of view, however, this estimation method belongs to primary fitting, and the algebraic accuracy is only one. To improve the accuracy of the curved edge area, piecewise linear fitting is usually used in mathematics. Figure $4 \mathrm{~b}$ shows a more accurate method in which each red segment is encrypted into 4 blue segments. The area of a curved edge is consequently replaced by the sum of the area of the $4 \times n$ small blue rectangle. The new method for calculating water vapor information in each layer using the Newton-Cotes numerical integration formula is proposed under such mathematical thoughts.

The specific application of the Newton-Cotes numerical integration formula is shown below. The small blue squares (e.g., $s_{1}, s_{2}, s_{3}, s_{4}, s_{5}$ ) in Figures $5 \mathrm{a}$ and $5 \mathrm{~b}$ are the interpolation points installed according to the Newton-Cotes formula (6) and (7). In this paper, contrary to the stratification interval of the lower layer (below $4 \mathrm{~km}$ ), the stratification interval of the upper layer (above $4 \mathrm{~km}$ ) is large. Thus, the Cotes formula (6) is applied to the lower layer where five interpolation points (including the two points at the two ends just in the layer) are installed in each layer, and the complex Cotes formula (7) is adopted at the upper layer where seventeen interpolation points are installed in each layer, as shown in Figures 5a and 5b. The formula for calculating the SWV value is transformed into the following expression:

$$
\begin{gathered}
S W V_{q}^{r}=\sum 10^{-6} \cdot \int_{i-1}^{i} \rho\left(s_{i}\right) d s_{i}= \\
=\sum 10^{-6} \cdot \sum_{i} \frac{s_{i}}{90}\left[7 f\left(s_{i}^{1}\right)+32 f\left(s_{i}^{2}\right)\right. \\
++12 f\left(s_{i}^{3}\right)+32 f\left(s_{i}^{4}\right) \\
\left.+7 f\left(s_{i}^{5}\right)\right]
\end{gathered}
$$




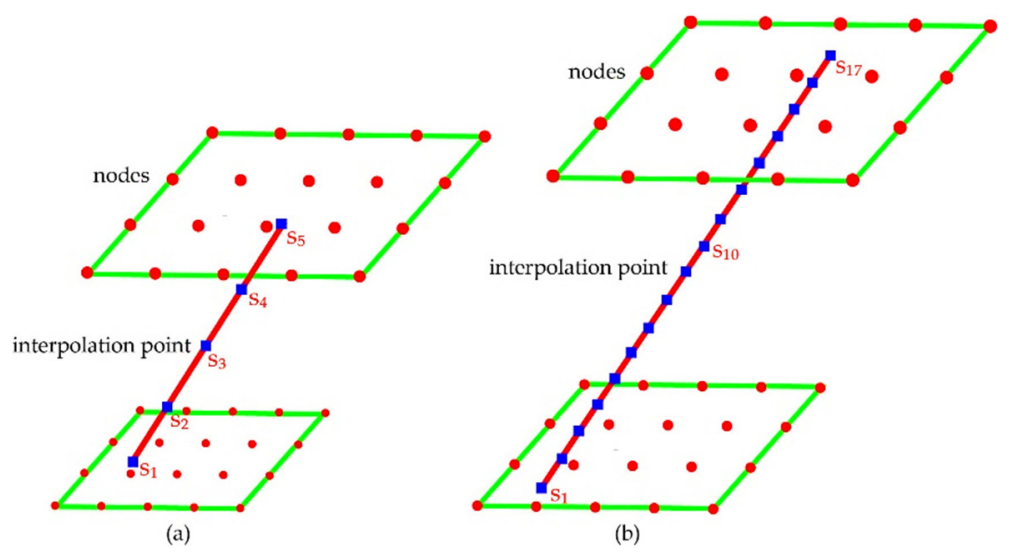

Fig. 5 The setting of interpolation points in the lower layer (a) and the upper layer (b). The small blue squares in both figures are interpolation points and the red dots represent the nodes in Figure $3 \mathrm{~b}$.

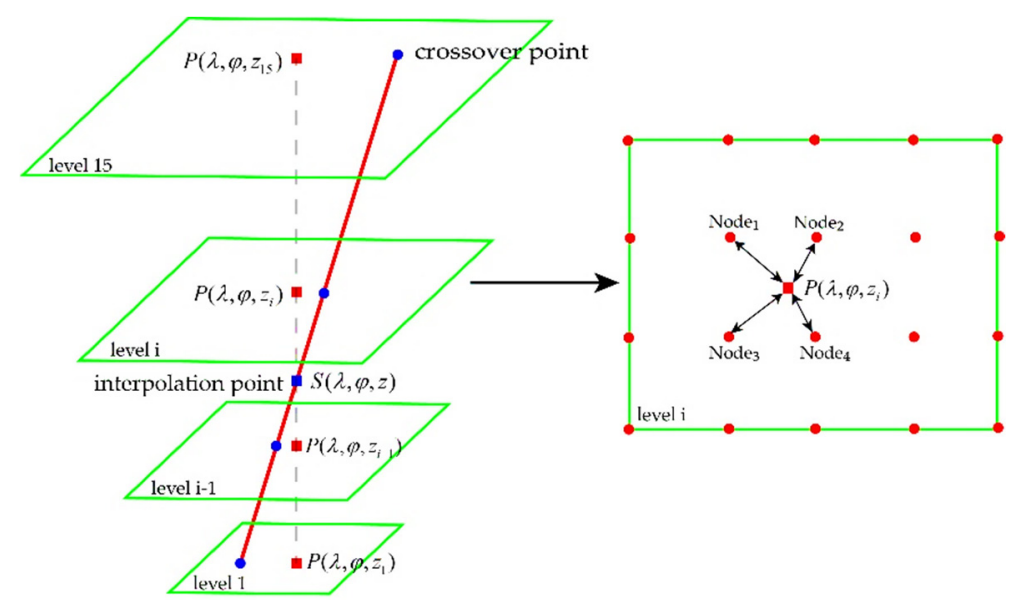

(a)

(b)

Fig. 6 Two step unification of the interpolation points and nodes. (a) The first step: computing the vertical projection coefficient between each interpolation point (small blue square) and its projection point (small red square) using the cubic spline interpolation. (b) The second step: horizontal weighting coefficients between projection points and their surrounding nodes are calculated by Gaussian weighting function.

where $\rho\left(s_{i}^{1}\right), \rho\left(s_{i}^{2}\right), \rho\left(s_{i}^{3}\right), \rho\left(s_{i}^{4}\right)$, and $\rho\left(s_{i}^{5}\right)$ represent the water vapor density at five interpolation points in Figure $3 \mathrm{a}$ and $s_{i}$ is the length of the signal passing through the $i$ th layer. Compared with Equation (8), Equation (9) has a higher calculation accuracy. For the sake of simplicity, the calculation of a high precision SWV value by the Cotes formula is listed here only. The application steps of complex Cotes formula are similar to the aforementioned.

\subsubsection{ESTABLISHING THE NUMERICAL RELATIONSHIP BEUREWEEN INTERPOLATION POINTS AND NODES}

According to Equation (9), approximately one hundred interpolation points are installed on each signal. In addition, the water vapor density at the interpolation point on each signal is different, thus thousands of unknowns appear in the set time interval (e.g., 30 minutes) when the water vapor density of interpolation points is regarded as an unknown parameter. Obviously, this is not feasible. Therefore, to improve the computational efficiency and accuracy of the node tomographic model, the new numerical relationship between water vapor density at the interpolation points (blue squares in Fig. 5) and water vapor density at the nodes (red nodes in Fig. 5) is established according to the spatial distribution characteristics of water vapor density. This part includes the following two steps, which are shown in Figures $6 \mathrm{a}$ and $6 \mathrm{~b}$.

As shown in Figure 5, the spatial location relationship between interpolation points and nodes is complex and irregular. Therefore, projection points are introduced to solve this problem in Figure 6a. Taking interpolation point $S(\lambda, \varphi, z)$ as an example, it is projected to each level along the vertical direction (gray dashed line in Fig. 7a), and the small red squares on each level in Figure 6a are the projection points (e.g., $\left.\mathrm{P}\left(\lambda, \varphi, z_{1}\right), \mathrm{P}\left(\lambda, \varphi, z_{i}\right), \cdots, \mathrm{P}\left(\lambda, \varphi, z_{i+1}\right), \cdots, \mathrm{P}\left(\lambda, \varphi, z_{15}\right)\right)$. 
The quantitative relationship between water vapor density at the interpolation point and water vapor density at the projection point is computed using the cubic spline interpolation commonly used in tropospheric atmospheric research $(\mathrm{Yu}$ et al., 2018a). The layered configuration consists of 15 planes in this paper, corresponding to 15 projection points. The water vapor density at the interpolation point $\mathrm{S}(\lambda, \varphi, z)$ can be calculated using the water vapor density at 15 projection points from $\mathrm{P}\left(\lambda, \varphi, z_{1}\right)$ to $\mathrm{P}\left(\lambda, \varphi, z_{15}\right)$ with the algorithm below.

$$
\begin{aligned}
\rho_{S(\lambda, \varphi, z)}=\frac{z_{i}-z}{\Delta z_{i}} \cdot \rho_{\mathrm{P}\left(\lambda, \varphi, z_{i-1}\right)}+\left(\frac{\left(z_{i}-z\right)^{3}}{6 \Delta z_{i}}-\frac{\Delta z_{i}\left(z_{i}-z\right)}{6}\right) \cdot D_{i-1,:} \times\left[\begin{array}{c}
\rho_{\mathrm{P}\left(\lambda, \varphi, z_{1}\right)} \\
\rho_{\mathrm{P}\left(\lambda, \varphi, z_{2}\right)} \\
\vdots \\
\rho_{\mathrm{P}\left(\lambda, \varphi, z_{i}\right)} \\
\vdots \\
\rho_{\mathrm{P}\left(\lambda, \varphi, z_{n}\right)}
\end{array}\right]+\quad \frac{z-z_{i-1}}{\Delta z_{i}} \cdot \rho_{\mathrm{P}\left(\lambda, \varphi, z_{i}\right)}+ \\
+\left(\frac{\left(z-z_{i-1}\right)^{3}}{6 \Delta z_{i}}-\frac{\Delta z_{i}\left(z-z_{i-1}\right)}{6}\right) \cdot D_{i,:} \times\left[\begin{array}{c}
\rho_{\mathrm{P}\left(\lambda, \varphi, z_{1}\right)} \\
\rho_{\mathrm{P}\left(\lambda, \varphi, z_{2}\right)} \\
\vdots \\
\rho_{\mathrm{P}\left(\lambda, \varphi, z_{i}\right)} \\
\vdots \\
\rho_{\mathrm{P}\left(\lambda, \varphi, z_{n}\right)}
\end{array}\right]
\end{aligned}
$$

Where $z_{i-1}$ and $z_{i}$ are the altitude of the two planes below and above the interpolation point, respectively, $\Delta z_{i}=z_{i}-z_{i-1}$ is the thickness of the $i$ th layer, $D_{i-1, \text { : }}$ and $D_{i, \text { : }}$ represent rows $i-1$ and $i$ of the matrix $\mathrm{D}$ where $\mathrm{D}=-A^{-1} B$. The calculations of $\mathrm{A}$ and $\mathrm{B}$ are expressed by Equation (11) and (12).

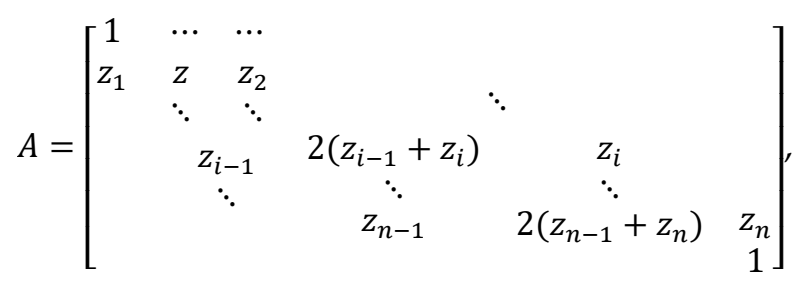

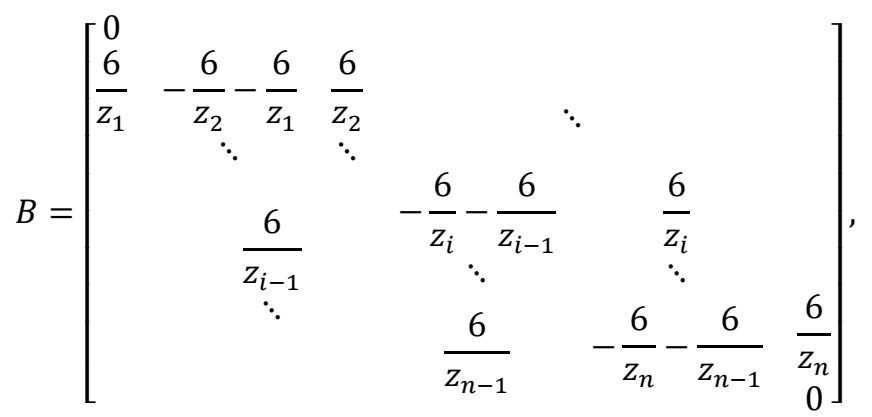

As $A$ and $B$ are only related to the altitude of each level, the matrix $D$ has to be computed once in the process of node parameterization. By introducing this algorithm, each interpolation point is associated with a projection point. The mission of calculating the water vapor density of the interpolation point is translated to compute the vapor density at the projection point, which is the first step to change.

Similarly, the second step is to establish the quantitative relationship between the projection point (small red square in Fig. 6b) and nodes (small red dots in Fig. 6b) in each layer. In the voxels model, the Gaussian weighting function is used as a smoothing constraint to represent the spatial characteristics of water vapor density in the horizontal direction (Song et al., 2006), which is introduced to calculate the weighting coefficients between projection points and their surrounding nodes in this study. Therefore, the vapor density at the projection point $\rho_{(\lambda, \varphi, z)}$ can be obtained by the following Equation:

$\rho_{(\lambda, \varphi, z)}=\frac{w_{1} \rho_{\left(\lambda_{1}, \varphi_{1}, z\right)}+w_{2} \rho_{\left(\lambda_{2}, \varphi_{2}, z\right)}+\cdots+w_{n} \rho_{\left(\lambda_{n}, \varphi_{n}, z\right)}}{n}=\sum_{i=1}^{n} w_{i} \rho_{\left(\lambda_{i}, \varphi_{i}, z\right)}$

where $w_{1}, w_{2}, \cdots, w_{n}$ are the weighting coefficients of the projection points, $\rho_{\left(\lambda_{1}, \varphi_{1}, z\right)}, \rho_{\left(\lambda_{2}, \varphi_{2}, z\right)}, \cdots, \rho_{\left(\lambda_{n}, \varphi_{n}, z\right)}$ represent water vapor density at the surrounding nodes, and $n$ is the number of the surrounding nodes, which is determined in the experiment. The weighting coefficients $w_{i}$ are expressed by:

$w_{i}=\frac{\exp \left(-\frac{d_{i}^{2}}{2 \sigma^{2}}\right)}{\sum_{i=1}^{n} \exp \left(-\frac{d_{i}^{2}}{2 \sigma^{2}}\right)}$, 
where $d_{i}$ indicates the distance between a projection point and the surrounding nodes, and $\sigma$ denotes a smoothing factor, which is influenced by the range of smoothing assumptions.

After the above procedure of node parameterization, a quantitative relationship is established between each Newton-Cotes interpolation points and the nodes in the 3-D tomographic field, which is essential for the construction of the tomographic observation equations.

\subsection{CONSTRUCTION AND SOLUTION OF TOMOGRAPHY OBSERVATION EQUATIONS} follows:

Based on Equation (9)-(14), the tomographic observation equation of a GNSS signal is constructed as

$$
\begin{aligned}
& S W V_{q}^{r}=10^{-6} \cdot\left[\int_{q}^{1} \rho\left(s_{1}\right) d s_{1}+\int_{1}^{2} \rho\left(s_{2}\right) d s_{2}+\cdots+\int_{i-1}^{i} \rho\left(s_{i}\right) d s_{i}+\cdots+\int_{n-1}^{n} \rho\left(s_{n}\right) d s_{n}\right]= \\
& =10^{-6} \cdot\left(\lambda_{11} \cdot \rho\left(x_{11}\right)+\lambda_{12} \cdot \rho\left(x_{12}\right)+\cdots+\lambda_{1 j} \cdot \rho\left(x_{1 j}\right)+\cdots+\lambda_{1 m} \cdot \rho\left(x_{1 m}\right)\right)+10^{-6} \cdot\left(\lambda_{21} \cdot\right. \\
& \left.\rho\left(x_{21}\right)+\lambda_{22} \cdot \rho\left(x_{22}\right)+\cdots+\lambda_{2 j} \cdot \rho\left(x_{2 j}\right)+\cdots+\lambda_{2 m} \cdot \rho\left(x_{2 m}\right)\right) \cdots+10^{-6} \cdot\left(\lambda_{i 1} \cdot \rho\left(x_{i 1}\right)+\lambda_{i 2} \cdot\right. \\
& \left.\rho\left(x_{i 2}\right)+\cdots+\lambda_{i j} \cdot \rho\left(x_{i j}\right)+\cdots+\lambda_{i m} \cdot \rho\left(x_{i m}\right)\right)+\cdots+10^{-6} \cdot\left(\lambda_{n 1} \cdot \rho\left(x_{n 1}\right)+\lambda_{n 2} \cdot \rho\left(x_{n 2}\right)+\cdots+\right. \\
& \left.\lambda_{n j} \cdot \rho\left(x_{n j}\right)+\cdots+\lambda_{n m} \cdot \rho\left(x_{n m}\right)\right) \text {, }
\end{aligned}
$$

where the coefficient of the observation equation consists of $\lambda_{11}-\lambda_{n m}, n$ is the number of layers and $m$ represents the number of nodes in each layer. $\rho_{\left(x_{11}\right)}-\rho_{\left(x_{n m}\right)}$ are the estimated parameters of water vapor density. In a tomographic epoch, multiple GNSS signals correspond to multiple observation equations, and a system of linear equations consisting of a large number of the above linear equations is shown below.

$$
\left[\begin{array}{c}
S W V_{1} \\
S W V_{2} \\
\vdots \\
S W V_{k}
\end{array}\right]=\left[\begin{array}{ccccccccc}
\lambda_{11}^{1} & \lambda_{12}^{1} & \cdots & \lambda_{1 m}^{1} & \cdots \cdots & \lambda_{n 1}^{1} & \lambda_{n 2}^{1} & \cdots & \lambda_{n m}^{1} \\
\lambda_{11}^{2} & \lambda_{12}^{2} & \cdots & \lambda_{1 m}^{2} & \cdots \cdots & \lambda_{n 1}^{2} & \lambda_{n 2}^{2} & \cdots & \lambda_{n m}^{2} \\
\vdots & \vdots & \ddots & \vdots & \cdots \cdots & \vdots & \vdots & \ddots & \vdots \\
\lambda_{11}^{k} & \lambda_{12}^{k} & \cdots & \lambda_{1 m}^{k} & \cdots \cdots & \lambda_{n 1}^{k} & \lambda_{n 2}^{k} & \cdots & \lambda_{n m}^{k}
\end{array}\right]\left[\begin{array}{c}
\rho\left(x_{11}\right) \\
\rho\left(x_{12}\right) \\
\vdots \\
\rho\left(x_{n m}\right)
\end{array}\right],
$$

For the tomographic observation Equation (16), the high-precision solving algorithm is also an important factor for ensuring the accuracy of the tomographic model. There are a few ways to solve the tomographic equations (Brenot et al., 2019). Singular Value Decomposition (SVD), the non-iterative approaches for solving the ill-condition system, is applied in some research ( Flores et al., 2000; Champollion et al., 2005; Song et al., 2006; Rohm, 2013). Gradinarsky and Jarlemark (2004) adopt the Kalman filter to retrieve the 3D distribution of the wet refractivity. In addition, the results of Heublein et al. (2019) show that the compressive sensing (CS) approach yields a more accurate and more precise solution than least squares (LSQ) in calculating the unknown parameters. Bender et al. (2010) analyze different algebraic reconstruction techniques (ART) that avoids the inversion of the normal equation by iteration. Moreover, this technology has been successfully applied to the reconstruction of a water vapor field in tropospheric tomography (Xiaoying et al., 2013; Prol et al., 2019). The iteration formula is shown below.

$x^{k+1}=x^{k}+\lambda \cdot \frac{m_{i}-\left\langle A^{i}, x^{k}\right\rangle}{\left\langle A^{i}, A^{i}\right\rangle} \cdot A^{i}$,

where $A$ indicates the coefficient matrix of Equation (16) and $A^{i}$ is the ith row of the matrix $A, m_{i}$ represents the column vectors on the left side of the Equation (16), and $\lambda$ denotes the relaxation factor which makes a great difference in the iteration results. It is noted that $\lambda$ can be adaptively adjusted according to the observation equations of different tomographic epochs in this paper.

\section{VERIFICATION OF THE IMPROVED TOMOGRAPHY APPROACH \\ 4.1. EXPERIMENTAL SCHEME}

GNSS observation data of 12 uniform distribution continuously operating reference stations were obtained from the Hong Kong Satellite Positioning Reference Station Network (SatRef). Figure 7 shows their locations (red dots) in the horizontal direction, and the blue star represents the location of the Radiosonde HKKP in Hong Kong. About the setting of vertical resolution, some researchers have investigated the effect of vertical layered configuration on the accuracy of tomographic models. Studies have indicated that a nonuniform stratified tomographic model has better accuracy (Rohm and Bosy, 2011; Chen and Liu, 2014; Ye et al., 2016), which is introduced in this work. The vertical resolution of the tomography region is shown in Figure 8, where the tomography region is segmented into 15 non-uniform layers from $0 \mathrm{~km}$ to $11000 \mathrm{~km}$ where the water vapor density is close to $0 \mathrm{~g} / \mathrm{m}^{3}$. 


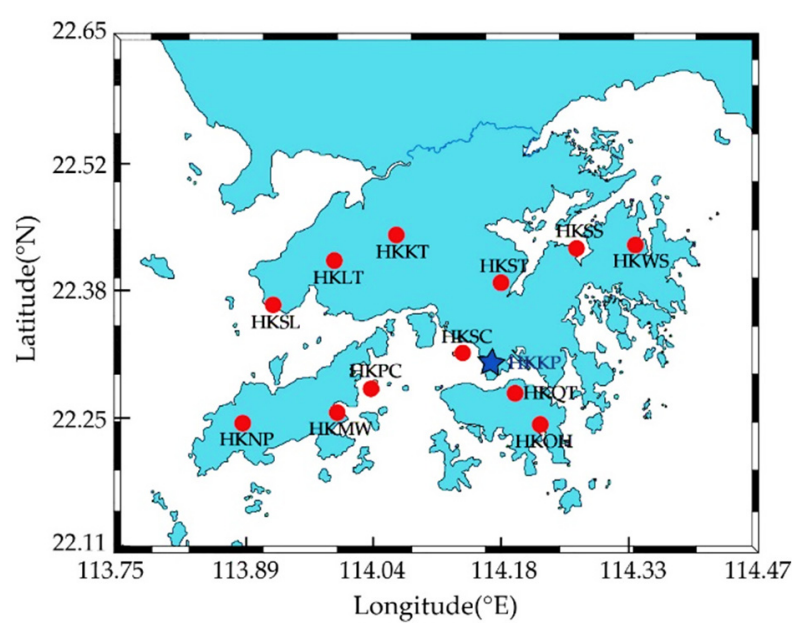

Fig. 7 Plane map of the Hong Kong area obtained by Mercator projection with 12 GNSS reference stations (red dots) and radiosonde HKKP (blue star) in Hong Kong.

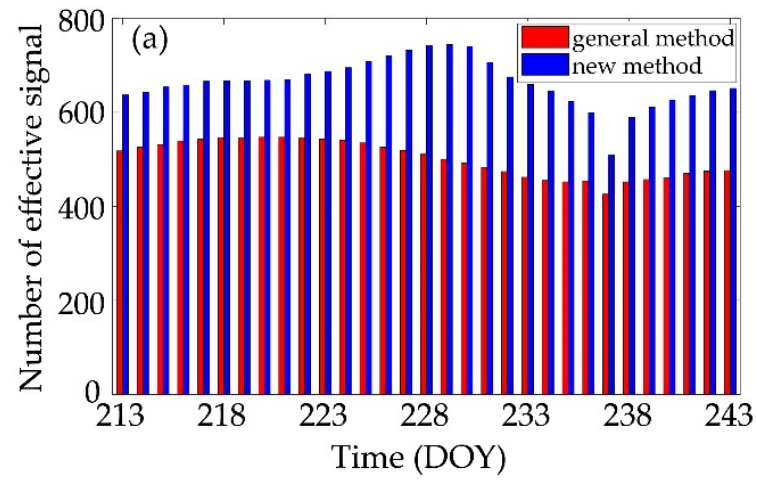

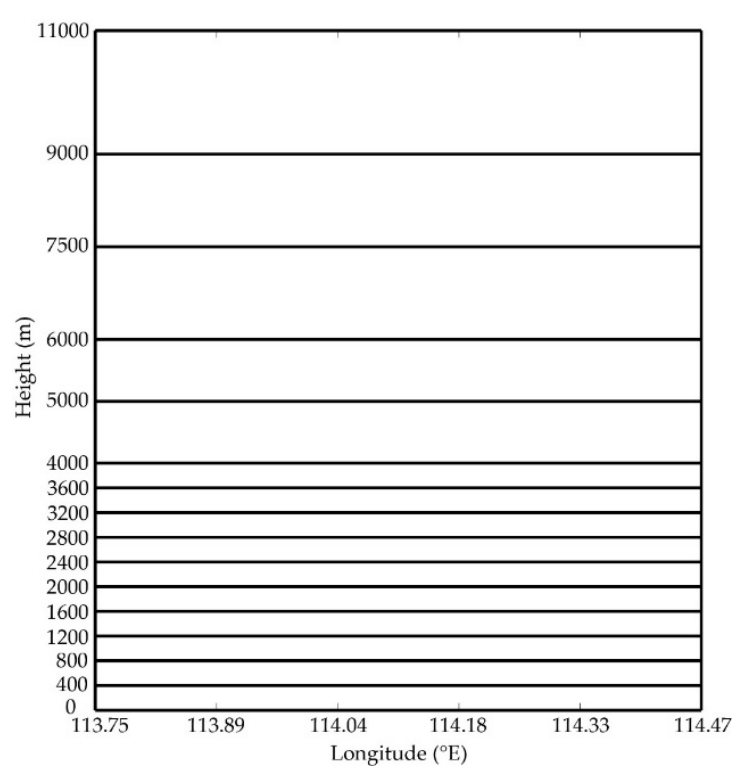

Fig. 8 Non-uniform vertical layer strategy for the tomographic model.

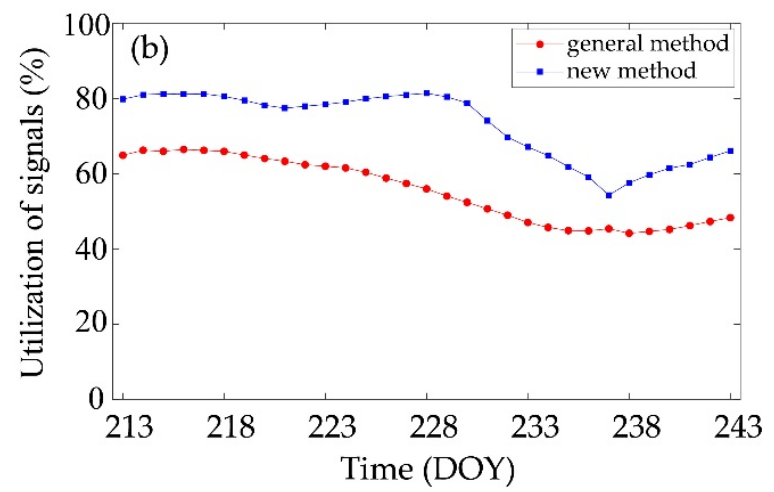

Fig. 9 The number of effective signals (a) and the utilization rate of signals (b) for general and new method during the period of DOY 213-243, 2017.

The GNSS observations from 12 GNSS reference stations and 3 IGS stations are preprocessed by GAMIT/GLOBK 10.6 version, which is the high precision software for GNSS baseline solution. The sampling rate of GNSS data is 30 seconds, while the time resolution of estimated ZWD and gradient delay is 5 minutes, which is sufficient to reflect the time variation characteristics of water vapor. The experimentation time is set in August 2017, that is, day of year (DOY) 213-243, when Hong Kong is in the summer, and there are more rainstorms. In addition, two special experiments were carried out to compare the tomographic accuracy of the new node model and the general voxel model on sunny and rainy days in Sect. 4.2.4.

\subsection{ANALYSIS OF THE IMPROVED TOMOGRAPHY METHOD}

To validate the performance of the proposed method, we made tomographic solutions using the general method and the improved approach, identified as Scheme 1 and Scheme 2, respectively. The two time periods of 00:00-00:30 UTC and 12:00-12:30 UTC per day serve as the time field of tomographic experiments, and tomographic results are compared with radiosonde data at 00:00 UTC and 12:00 UTC due to its ability to obtain accurate water vapor density profiles at different altitudes.

\subsubsection{NUMBER OF EFFECTIVE SIGNALS AND SIMILARITY}

In the following, Figure 9 shows the analysis of the number of effective signals and utilization rate. The statistical result over the experimental period shows that, when the elevation angle greater than $15^{\circ}$ instead of penetrating from the tomography top is considered as criterion, the average effective signals is increased by $32.33 \%$, whereas the average utilization rate of signals is enhanced by $12.62 \%$ from $55.37 \%$ to $72.19 \%$. Additionally, the stable similarity varying 


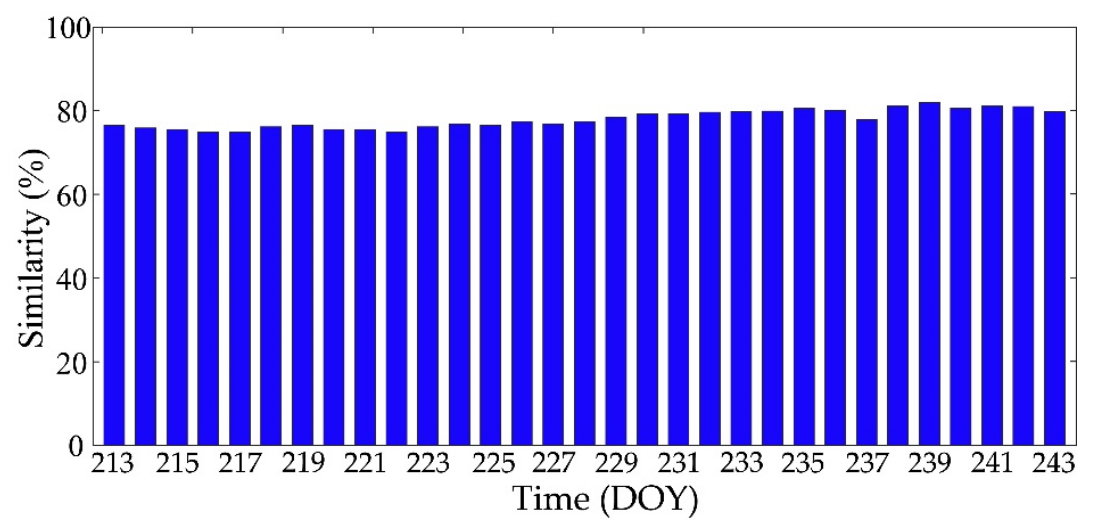

Fig. 10 The similarity of convex hull boundary and rectangle one during the tomographic period.
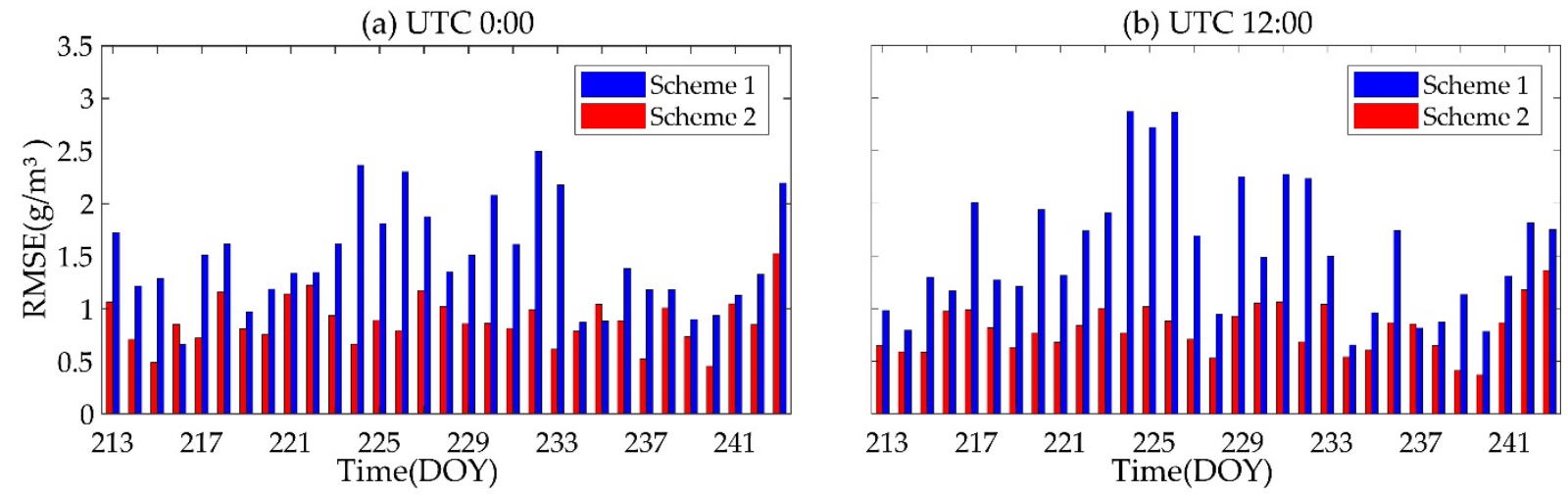

Fig. 11 Comparisons between the tomography results of the Scheme 1 (blue) and Scheme 2 (red) during the 31-day period from DOY 213 to DOY 243, 2017, at UTC 00:00 (a) and UTC12:00 (b) daily.

from $75 \%$ to $80 \%$ can be found in Figure 10, which shows that the proposed optimal rectangular boundaries are useful and reliable.

\subsubsection{TOMOGRAPHIC RESULTS OF SCHEME 1 AND SCHEME 2}

The tomographic results from two schemes are interpolated to calculate the water vapor density at the position of the radiosonde station in each layer. The RMSE (root-mean-square error) between the two tomographic results and the radiosonde data are shown in Figure 11, which refers to the 31-day period from DOY 213 to DOY 243, 2017, at 00:00 and 12:00 UTC daily. Besides, Table 1 lists the statistics including the maximum, minimum and mean values of Bias, STD (standard deviation) and RMSE.

It should be noted that Scheme 2 has a smaller RMSE than Scheme 1 in a majority of time periods, and the accuracy of some epochs is increased by $70 \%$, e.g., the value of RMSE decreased from $2.5020 \mathrm{~g} / \mathrm{m}^{3}$ to $0.6832 \mathrm{~g} / \mathrm{m}^{3}$ at DOY 225 UTC 0:00, when the accuracy has been improved by $73.41 \%$. Additionally, there are 22 days in which the accuracy improvement rate is more than $30 \%, 11$ of them increased by more than $50 \%$. Further, it can be seen that Scheme 2 significantly outperformed Scheme 1 in all three statistics from Table 1, except for the minimum value of Bias. However, the bias distribution of the Scheme 2 is more centralized and balanced than that of the Scheme 1 from Figure 12. Further, Figure 13 denotes the distribution of two tomographic schemes, and a comprehensive comparison in Figure 13c shows the consistent conclusion that the Scheme 2 provided more accurate tomographic results than Scheme 1.

\subsubsection{ACCURACY ANALYSIS OF EACH LAYER}

In this subsection, more refined errors in each of the tomographic layers are compared. The comparison of the RMSE and relative error of every layer between water vapor density derived from two schemes are shown in Figure 14.

It is well known that the RMSE value decreases with the increase of height as the water vapor density in the lower layer is much higher than that in the upper layer. It can be observed from Figure 14a that the differences obtained by scheme 1 are small than those provided by scheme 2, particularly in the lower layers from 0 to $2 \mathrm{~km}$. In this altitude range, plenty of water 
Table 1 Statistics (Bias, STD, RMSE) of the tomography results for two schemes. unit: $\mathrm{g} / \mathrm{m}^{3}$.

\begin{tabular}{lllllllllr}
\hline \multirow{2}{*}{ Scheme } & Bias & \multicolumn{3}{c}{ STD } & \multicolumn{3}{c}{ RMSE } \\
\cline { 2 - 10 } & Max. & Min. & Mean & Max. & Min. & Mean & Max. & Min. & \multicolumn{1}{c}{ Mean } \\
\hline Scheme 1 & 0.98 & 0.50 & 0.36 & 2.97 & 0.52 & 1.47 & 2.87 & 0.65 & 1.51 \\
Scheme 2 & 0.75 & 0.74 & 0.15 & 1.16 & 0.38 & 0.77 & 1.22 & 0.40 & 0.83 \\
\hline
\end{tabular}
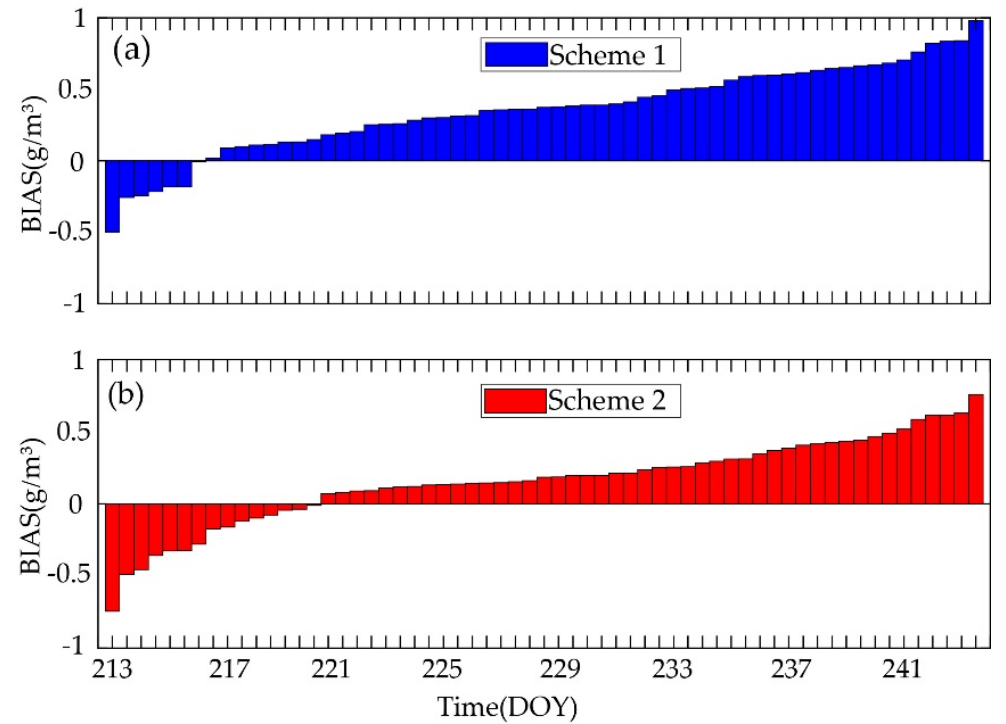

Fig. 12 Bias distribution of the tomographic results of the Scheme 1 (a) and the Scheme 2 (b).
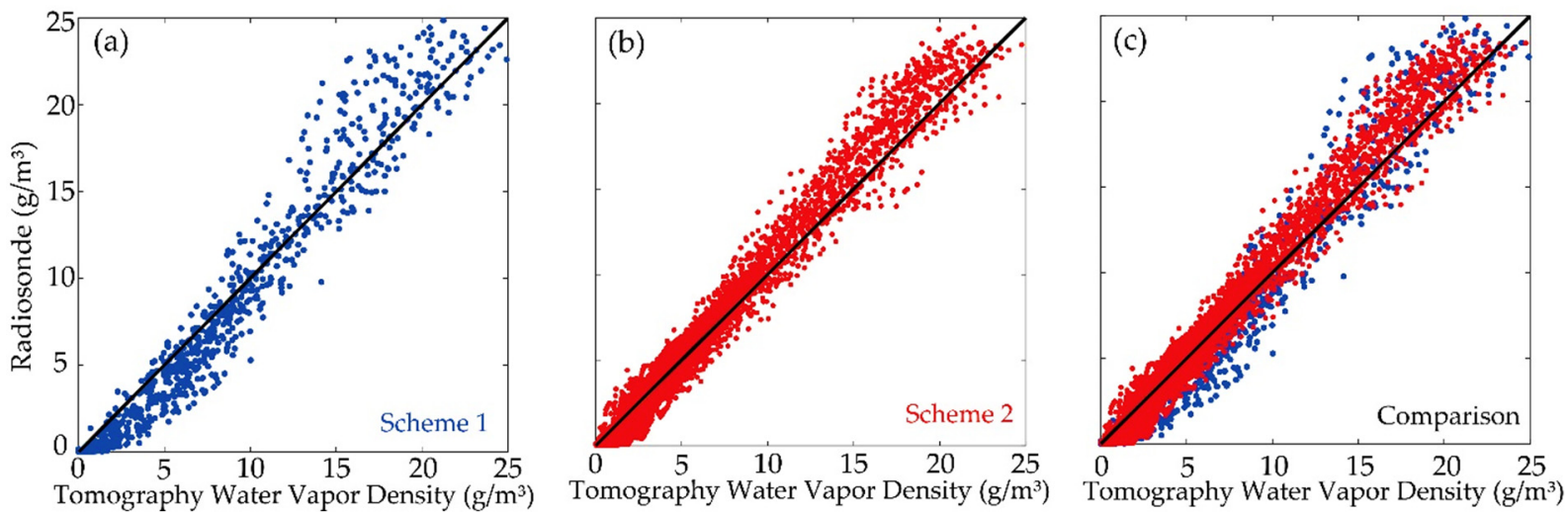

Fig. 13 Distribution of one-month Scheme 1 (a) and Scheme 2 (b) tomographic results compared with the radiosonde data and comprehensive comparison (c).

vapor, probably accounting for $60 \%$ of the total, is concentrated and variable. These results also validate the superiority of the improved tomography method in this work.

In addition, it should be noted that the relative error dramatically increases with the increase of height above $5 \mathrm{~km}$ in Figure 14b, and especially is greater than $100 \%$ above $6 \mathrm{~km}$ to $9 \mathrm{~km}$. This phenomenon is not a calculation error, as water vapor mainly distributes below $5 \mathrm{~km}$ near the earth's surface, and water vapor content above $6 \mathrm{~km}$ is so low that it is below the corresponding RMSE value. Figures $15 \mathrm{a}$ and $15 \mathrm{~b}$ show the box statistical diagram for the errors of the two schemes. A similar finding is indicated in Figure 15, where the tomographic results of Scheme 2 are more centralized and stable than those of Scheme 1 .

\subsubsection{ACCURACY COMPARISON UNDER DIFFERENT WEATHER CONDITIONS}

To further compare the accuracy of the two tomographic methods, two-day observations were used for tomographic experiments. One is DOY 228 when Hong Kong was sunny, the other is DOY 239 when Hong Kong suffered heavy rain. As radiosonde can only provide observed data at 0:00 UTC and 12:00 

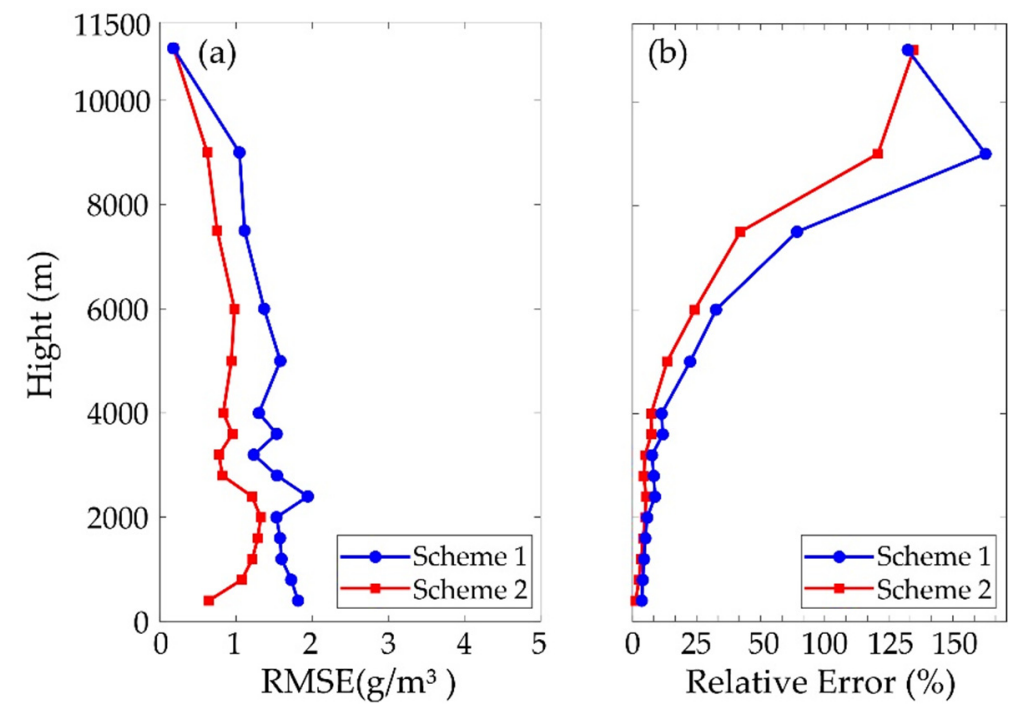

Fig. 14 RMSE (a) and relative error (b) at each layer of the tomographic results from the Scheme 1 (blue curve) and Scheme 2 (red curve).
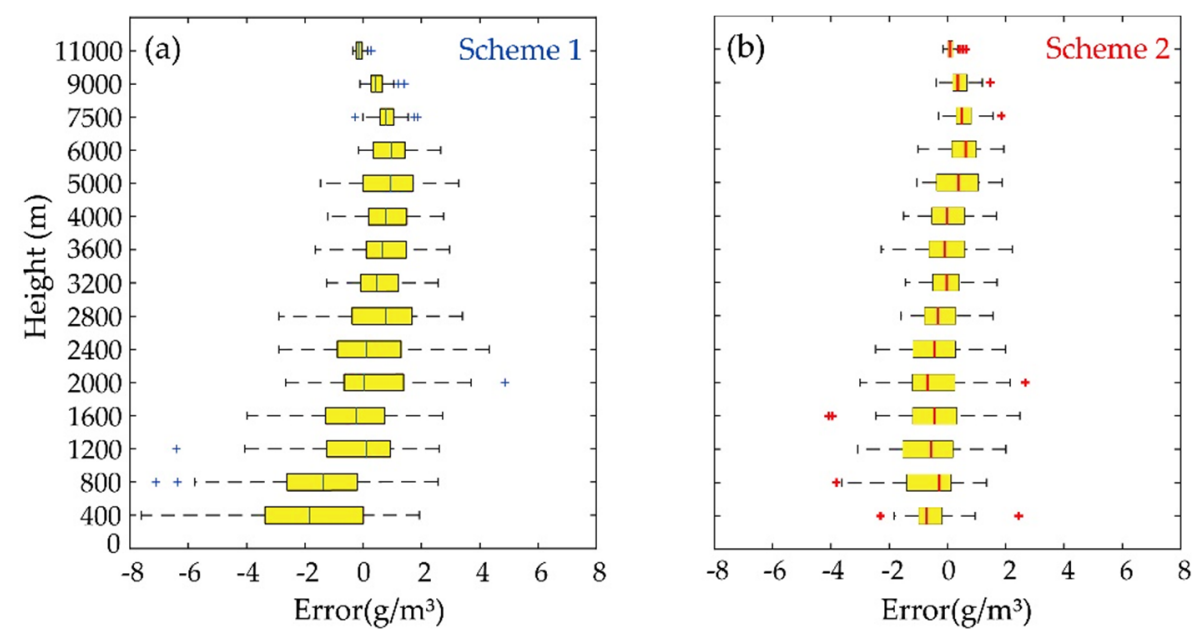

Fig. 15 Box plot of the error between the radiosonde data and tomographic results from Scheme 1 (a) and Scheme 2 (b).

UTC daily, only the tomographic results at these twotime points are evaluated. Table 2 lists the weather conditions at these days.

Water vapor profiles under two meteorological conditions are shown in Figure 16. It is obvious that the tomographic results of Scheme 2 match better with the radiosonde data than Scheme 1, and the coincidence is higher on rainy days.

Comparing the results over the two days, the vertical distributions of water vapor are more even during the rainy day than those during the sunny day. On the fine day, water vapor mainly concentrates below $4 \mathrm{~km}$ altitude, where the water vapor density is more than $5 \mathrm{~g} / \mathrm{m}^{3}$ and near $0 \mathrm{~g} / \mathrm{m}^{3}$ above $6 \mathrm{~km}$ in altitude. However, the water vapor density is greater than $5 \mathrm{~g} / \mathrm{m}^{3}$ below $6 \mathrm{~km}$ in altitude on a rainy day and near $0 \mathrm{~g} / \mathrm{m}^{3}$ above $8 \mathrm{~km}$ in altitude. In addition, it is critical to note that the tomographic results from the rainy day are much better than those from the sunny day for both schemes at heights of 0 to $1 \mathrm{~km}$. These good tomographic results also provide a basis for the forecast and analysis of rainstorm weather.

Table 3 shows statistics of the two tomographic schemes under two weather conditions. In both periods, the tomographic results of Scheme 2 have better accuracy than Scheme 1. The RMSE is reduced from $1.15 \mathrm{~g} / \mathrm{m}^{3}$ (Scheme 1) to $0.84 \mathrm{~g} / \mathrm{m}^{3}$ (Scheme 2) on a sunny day, and the accuracy is improved by $26.96 \%$, while on a rainy day, the accuracy is improved by $44.12 \%$ as the RMSE is decreased from $1.02 \mathrm{~g} / \mathrm{m}^{3}$ (Scheme 1) to $0.57 \mathrm{~g} / \mathrm{m}^{3}$ (Scheme 2), which indicates that the optimized method has a better improvement effect on rainy days than on sunny days.

\section{CONCLUSION}

An improved water vapor tomography method is proposed to construct a 3D tomographic water vapor field, which showed its superiority over the general 
Table 2 Weather conditions at UTC 0:00 and UTC 12:00 on DOY 226 and DOY 239.

\begin{tabular}{lrllc}
\hline DOY & UTC & Weather & Wind Direction & Temperature $\left({ }^{\circ} \mathbf{C}\right)$ \\
\hline \multirow{2}{*}{228} & 0 & cloudy & no sustained & 29 \\
& 12 & fine & no sustained & 33 \\
239 & 0 & rainstorm & north & 25 \\
& 12 & rainstorm & southeast & 30 \\
\hline
\end{tabular}

(a) DOY 228 UTC 0

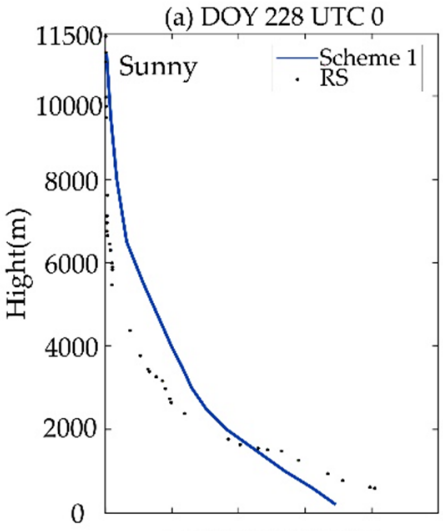

(e) DOY 228 UTC 0

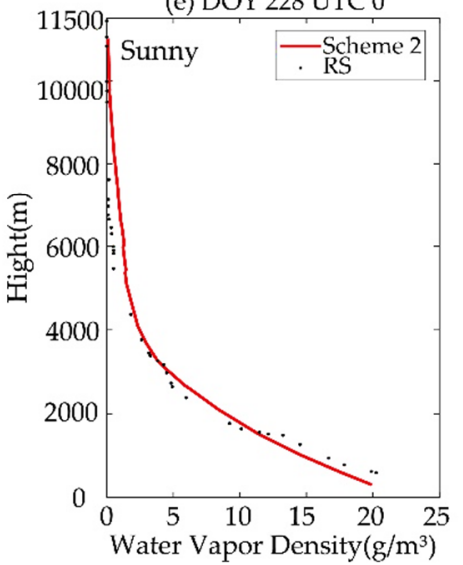

(b) DOY 228 UTC 12

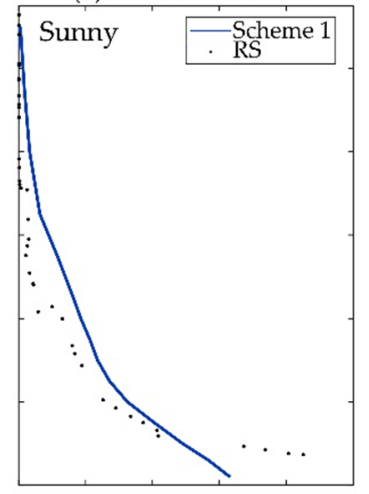

(f) DOY 228 UTC 12

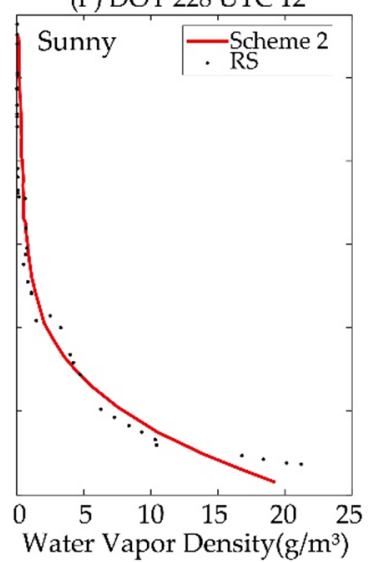

(c) DOY 239 UTC 0

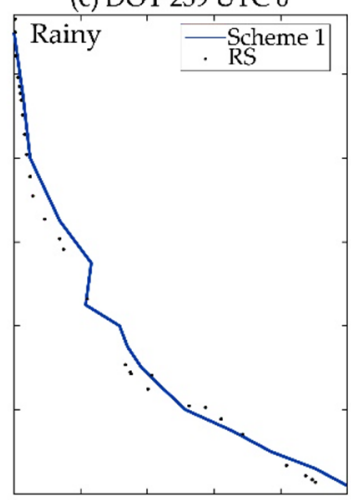

(g) DOY 239 UTC 0

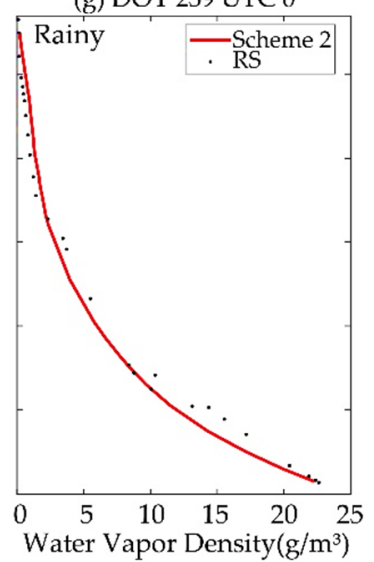

(d) DOY 239 UTC 12

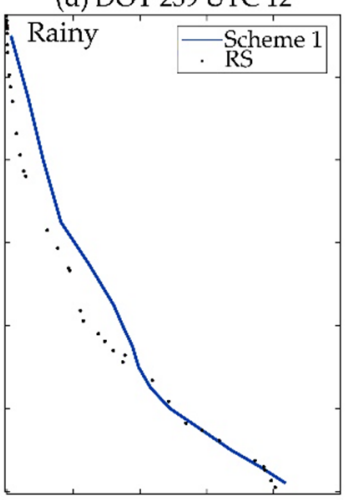

(h) DOY 239 UTC 12

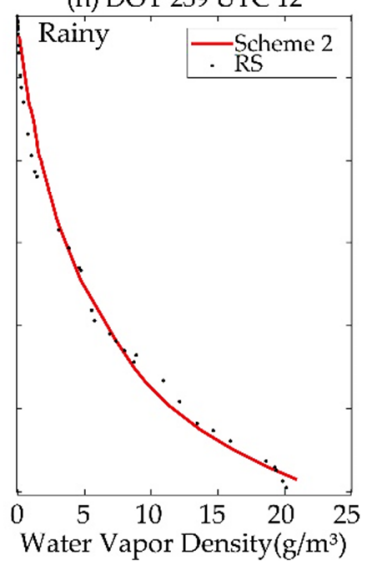

Fig. 16 Comparison of tomographic water vapor profiles between the Scheme 1 (a-d) and the Scheme 2 (e-h) under different weather conditions using the radiosonde (black dots) as a reference.

approach. The main improvements are described as follows: 1) Compared with the common tomography model, the optimized model is more flexible and suitable for the spatial distribution characteristics of satellite signals. Besides, more GNSS signals and broader tomographic scope are determined by the dynamic algorithm. 2) The node discretization method replaces the voxel discretization method, which has a higher conformity to the spatial distribution of water vapor and minimizes the discretization effects; and 3) Piecewise linear fitting is used to calculate the approximate value of SWV, which has higher accuracy compared with the common one-time fitting.

Two tomographic schemes based on the traditional and improved method were implemented, and the same GNSS observations during the period of 1-31 August 2017 from Hong Kong SatRef were processed in both tests. Radiosonde data from HKKP were used as reference values to evaluate the tomographic performance of the two ways. By comparing the statistics of the tomographic results, the analysis from different perspectives is as follows: On the one hand, the mean RMSE for 31 days was reduced from $1.5 \mathrm{~g} / \mathrm{m}^{3}$ with Scheme 1 to $0.83 \mathrm{~g} / \mathrm{m}^{3}$ with Scheme 2. Furthermore, the optimized mean shows superior performance compared to the common approach at each layer, especially in the lower layer of 0-4 km. On the other hand, two sub-experiments were carried out to analyze the improvement effect of the improved method under different weather conditions. 
Table 3 Statistical results of Node and Voxel tomographic water vapor density on a fine day and a rainy day. unit: $\mathrm{g} / \mathrm{m}^{3}$.

\begin{tabular}{llccc}
\hline Weather & Statistic & Scheme 1 & Scheme 2 & Accuracy improvement \\
\hline Sunny & Bias & 0.42 & 0.37 & $11.90 \%$ \\
& STD & 1.10 & 0.74 & $32.73 \%$ \\
& RMSE & 1.15 & 0.84 & $26.96 \%$ \\
& IQR & 1.41 & 1.07 & $24.11 \%$ \\
\hline Rainy & Bias & 0.54 & 0.24 & $55.56 \%$ \\
& STD & 0.85 & 0.52 & $38.82 \%$ \\
& RMSE & 1.02 & 0.57 & $44.12 \%$ \\
& IQR & 1.14 & 0.67 & $41.23 \%$ \\
\hline
\end{tabular}

In terms of the RMSE of the tomographic results, the accuracy was improved by $26.96 \%$ on a fine day while the accuracy improvement rate is $44.12 \%$ on a rainy day. In summary, all the performed validations showed the advantage of the optimized method for the enhancement of tomographic results.

Further investigations should focus on whether the improved method can improve the overall accuracy of the middle and boundary tomographic area. The tomographic boundary area with less signal passing will be more conducive to the analysis and study of water vapor changes in the whole region under some extreme weather conditions. A second point to investigate is the spare GNSS station network where there are too low station density to construct accurate 3D water vapor tomographic fields using the general method. However, the optimized method has unique advantages in this respect, which have yet to be implemented and verified.

\section{ACKNOWLEDGMENTS}

This study is supported by the National Natural Science Foundation of China (Grant 41774026 and Grant 41904013). The authors acknowledge the support of the Survey and Mapping Office (SMO) of Lands Department, HongKong, for provision of the SatRef GNSS data and the ground-based meteorological data. The King's Park Observatory is also acknowledged for providing the high-precision radiosonde data. The GAMIT/GLOBK software is provided by the Department of Earth Atmospheric and Planetary Sciences, MIT. The manuscript is polished by American Journal Experts (AJE). The authors would like to thank anonymous reviewers for the review and suggest of this paper.

\section{REFERENCES}

Alber, C., Ware, R., Rocken, C. and Braun, J.: 2000, Obtaining single path phase delays from GPS double differences. Geophys. Res. Lett., 27, 17, 2661-2664. DOI: $10.1029 / 2000 \mathrm{~g} 1011525$

Andersson, E., Hólm, E., Bauer, P., Beljaars, A., Kelly, G., McNally, A. et al.: 2007, Analysis and forecast impact of the main humidity observing system. Q. J. Roy Meteor. Soc., 133, 627, 1473-1485. DOI: $10.1002 /$ qj. 112

Bender, M., Dick, G., Ge, M., Deng, Z., Wickert, J., Kahle, H.-G. et al.: 2010, Development of a GNSS water vapour tomography system using algebraic reconstruction techniques. Adv. Space Res., 47, 10, 1704-1720. DOI: 10.1016/j.asr.2010.05.034

Benevides, P., Catalao, J. and Miranda, P.M.A.: 2015, On the inclusion of GPS precipitable water vapour in the nowcasting of rainfall. Nat. Hazards Earth Sys. Sci., $15,12,2605-2616$. DOI: $10.5194 /$ nhess-15-2605-2015

Benevides, P., Catalao, J., Nico, G. and Miranda, P.M.A.: 2018, 4D wet refractivity estimation in the atmosphere using GNSS tomography initialized by radiosonde and AIRS measurements: results from a 1-week intensive campaign. GPS Solut., 22, 4, 91. DOI:10.1007/s10291-018-0755-5

Benevides, P., Nico, G., Catalao, J. and Miranda, P.M.A.: 2016, Bridging InSAR and GPS tmography: A new differential geometrical constraint. IEEE Trans. Geosci. Remote Sens., 54, 2, 697-702. DOI: $10.1109 /$ tgrs. 2015.2463263

Benevides, P., Nico, G., Catalao, J. and Miranda, P.M.A.: 2017, Analysis of Galileo and GPS integration for GNSS tomography. IEEE Trans. Geosci. Remote Sens., 55, 4, 1936-1943. DOI: $10.1109 /$ tgrs. 2016.2631449

Bevis, M., Businge, S. and Herring, T.: 1992,. GPS meteorology: Remote sensing of atmospheric water vapor using global positioning system. J. Geophys. Res. Atmos., 97,D14, 15789-15801.

Boehm, J. and Schuh, H.: 2004, Vienna mapping functions in VLBI analyses. Geophys. Res. Lett., 31, 1, L01603. DOI: 10.1029/2003GL018984

Brenot, H., Rohm, W., Kacmařik, M., Moller, G., Sa, A., Tondaś, D. et al.: 2019, Cross-comparison and methodological improvement in GPS tomography. Remote Sens., 12, 1, 30. DOI: 10.3390/rs 12010030

Champollion, C., Masson, F., Bouin, M., Walpersdorf, A., Doerflinger, E., Bock, O. et al.: 2005, GPS water vapour tomography: Preliminary results from the ESCOMPTE field experiment. Atmos. Res., 74, 1, 253-274. DOI: 10.1016/j.atmosres.2004.04.003

Chen, B.Y. and Liu, Z.Z.: 2014, Voxel-optimized regional water vapor tomography and comparison with radiosonde and numerical weather model. J. Geod., 88, 7, 691-703. DOI: 10.1007/s00190-014-0715-y

Chen, B.Y., Liu, Z.Z., Wong, W.K. and Woo, W.C.: 2017, Detecting water vapor variability during heavy precipitation events in Hong Kong using the GPS tomographic technique. J. Atmos. Ocean. Technol., 34, 5, 1001-1019. DOI: 10.1175/Jtech-D-16-0115.1

Ding, N., Zhang, S., Wu, S., Wang, X., Kealy, A., Zhang, K.: 2018a, A new approach for GNSS tomography from a few GNSS stations. Atmos. Meas. Tech., 11, 6, 3511-3522. DOI: 10.5194/amt-11-3511-2018 
Ding, N., Zhang, S. and Zhang, Q.: 2017, New parameterized model for GPS water vapor tomography. Ann. Geophys., 35, 2, 311-323. DOI: 10.5194/angeo-35-311-2017

Ding, N., Zhang, S.B., Wu, S.Q., Wang, X.M. and Zhang, K.F.: 2018b, Adaptive node parameterization for dynamic determination of boundaries and nodes of GNSS tomographic models. J. Geophys. Res., Atmospheres, 123, 4, 1990-2003. DOI: $10.1002 / 2017 \mathrm{jd} 027748$

Dong, Z. and Jin, S.: 2018, 3-D Water vapor tomography in Wuhan from GPS, BDS and GLONASS observations. Remote Sens., 10, 2. DOI: 10.3390/rs10010062

dos Santos Prol, F., de Oliveira Camargo, P., HernandezPajares, M. and de Assis Honorato Muella, M.T.: 2019, A new method for ionospheric tomography and its assessment by ionosonde electron density, GPS TEC, and single-frequency PPP. IEEE Trans. Geosc. Remote Sens., 57, 5, 2571-2582. DOI: $10.1109 /$ tgrs.2018.2874974

Flores, A., De Arellano, J.V.G., Gradinarsky, L.P. and Rius, A.: 2001, Tomography of the lower troposphere using a small dense network of GPS receivers. IEEE Trans. Geosci. Remote Sens., 39, 2, 439-447.

Flores, A., Ruffini, G. and Rius, A.: 2000, 4D tropospheric tomography using GPS slant wet delays. Ann. Geophys., 18, 223-234.

DOI: $10.1007 / \mathrm{s} 00585-000-0223-7$

Gradinarsky, L. and Jarlemark, P.: 2004, Ground-based GPS tomography of water vapor: Analysis of simulated and real data. J. Meteorol. Soc. JPN, 82, 1, 551-560.

Graham, R.L.: 1972, An efficient algorithm for determining the convex hull of a finite planar set. Inform. Process. Lett., 1, 4, 132-133.

Heublein, M., Alshawaf, F., Erdnüß, B., Zhu, X.X. and Hinz, S.: 2019, Compressive sensing reconstruction of 3D wet refractivity based on GNSS and InSAR observations. J. Geod., 93, 2, 197-217. DOI: $10.1007 / \mathrm{s} 00190-018-1152-0$

Jaberi Shafei, M. and Mashhadi-Hossainali, M.: 2018, Application of the GNSS-R in tomographic sounding of the Earth atmosphere. Adv. Space Res., 62, 1, 7183. DOI: 10.1016/j.asr.2018.04.003

Lu, C., Li, X., Nilsson, T., Ning, T., Heinkelmann, R., Ge, M. et al.: 2015, Real-time retrieval of precipitable water vapor from GPS and BeiDou observations. J. Geod., 89, 9, 843-856.

DOI: $10.1007 / \mathrm{s} 00190-015-0818-0$

Möller, G. and Landskron, D.: 2019, Atmospheric bending effects in GNSS tomography. Atmos. Meas. Tech., 12, 1, 23-34. DOI: 10.5194/amt-12-23-2019

Perler, D., Geiger, A. and Hurter, F.: 2011, 4D GPS water vapor tomography: new parameterized approaches. J. Geod., 85, 8, 539-550.

DOI: $10.1007 / \mathrm{s} 00190-011-0454-2$

Rohm, W.: 2013, The ground GNSS tomography unconstrained approach. Adv. Space Res., 51, 3, 501513. DOI: 10.1016/j.asr.2012.09.021

Rohm, W. and Bosy, J.: 2011, The verification of GNSS tropospheric tomography model in a mountainous area. Adv. Space Res., 47, 10, 1721-1730. DOI: 10.1016/j.asr.2010.04.017

Saastamoinen, J.: 1972, Contributions to the theory of atmospheric refraction. J. Geod., 105, 1, 279-298.

Song, S., Zhu, W., Ding, J. and Peng, J.: 2006, 3D watervapor tomography with Shanghai GPS network to improve forecasted moisture field. Chin. Sci. Bull., 51, 607-614. DOI: 10.1007/s11434-006-0607-5
Wang, X., Zhang, K., Wu, S., Li, Z., Cheng, Y., Li, L. et al.: 2018, The correlation between GNSS-derived precipitable water vapor and sea surface temperature and its responses to El Niño-Southern Oscillation. Remote Sens. Environ., 216, 1-12.

DOI: 10.1016/j.rse.2018.06.029

Xiaoying, W., Ziqiang, D., Enhong, Z., Fuyang, K.E., Yunchang, C. and Lianchun, S.: 2013, Tropospheric wet refractivity tomography using multiplicative algebraic reconstruction technique. Adv. Space Res., 53, 1, 156-162. DOI: 10.1016/j.asr.2013.10.012

Yang, F., Guo, J., Shi, J., Zhao, Y., Zhou, L. and Song, S.: 2019, A new method of GPS water vapor tomography for maximizing the use of signal rays. Appl. Sci., 9, 7 , 1446. DOI: $10.3390 /$ app 9071446

Yao, Y. and Zhao, Q.: 2016, Maximally using GPS observation for water vapor tomography. IEEE Trans. Geosci. Remote Sens., 54, 12, 7185-7196. DOI: $10.1109 /$ tgrs. 2016.2597241

Ye, S.R., Xia, P.F. and Cai, C.S.: 2016, Optimization of GPS water vapor tomography technique with radiosonde and COSMIC historical data. Ann. Geophys., 34, 9, 789-799. DOI: 10.5194/angeo-34-789-2016

Yu, C., Li, Z., Penna, N.T. and Crippa, P.: 2018a, Generic atmospheric correction model for interferometric synthetic aperture radar observations. J. Geophys. Res., Solid Earth, 123, 10, 9202-9222. DOI: 10.1029/2017jb015305

Yu, W., Chen, B., Dai, W. and Luo, X.: 2018b, Real-time precise point positioning using tomographic wet refractivity fields. Remote Sens., 10, 6, 928. DOI: $10.3390 /$ rs 10060928

Zhao, Q., Yao, Y., Cao, X. and Yao, W.: 2019a, Accuracy and reliability of tropospheric wet refractivity tomography with GPS, BDS, and GLONASS observations. Adv. Space Res., 63, 9, 2836-2847. DOI: 10.1016/j.asr.2018.01.021

Zhao, Q., Yao, Y., Cao, X., Zhou, F. and Xia, P.: 2018a, An optimal tropospheric tomography method based on the multi-GNSS observations. Remote Sens., 10, 2, 234. DOI: $10.3390 / \mathrm{rs} 10020234$

Zhao, Q., Yao, Y. and Yao, W.: 2018b, Troposphere water vapour tomography: A horizontal parameterised approach. Remote Sens., 10, 8, 1241. DOI: $10.3390 / \mathrm{rs} 10081241$

Zhao, Q., Yao, Y., Yao, W. and Xia, P.: 2018c, An optimal tropospheric tomography approach with the support of an auxiliary area. Ann. Geophys., 36, 4, 1037-1046. DOI: 10.5194/angeo-36-1037-2018

Zhao, Q., Zhang, K. and Yao, W.: 2019b, Influence of station density and multi-constellation GNSS observations on troposphere tomography. Ann. Geophys., 37, 1, 15-24. DOI: 10.5194/angeo-37-15-2019

Zumberge, J.F., Heflin, M.B., Jefferson, D.C., Watkins, M.M. and Webb, F.H.: 1997, Precise point positioning for the efficient and robust analysis of GPS data from large networks. J. Geophys. Res., Solid Earth, 102, B3, 5005-5017. DOI: 10.1029/96jb03860

Zus, F.: 2018, Estimating the impact of global navigation satellite system horizontal delay gradients in variational data assimilation. Remote Sens.. DOI: $10.3390 /$ rs11010041 